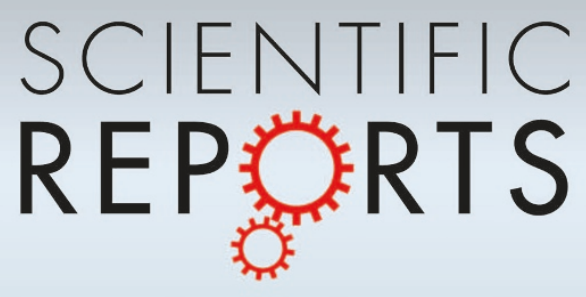

OPEN

SUBJECT AREAS:

SOLAR CELLS

ELECTRICAL AND ELECTRONIC

ENGINEERING

ELECTRONIC DEVICES

QUANTUM DOTS

Received

15 April 2013

Accepted

24 June 2013

Published

19 July 2013

Correspondence and requests for materials should be addressed to W.Y. (woojun.yoon@ ieee.org)

\section{Enhanced Open-Circuit Voltage of PbS Nanocrystal Quantum Dot Solar Cells}

\author{
Woojun Yoon' ${ }^{1}$ Janice E. Boercker', Matthew P. Lumb ${ }^{1,2}$, Diogenes Placencia' ${ }^{1}$ Edward E. Foos' \\ \& Joseph G. Tischler'
}

'U.S. Naval Research Laboratory, 4555 Overlook Avenue SW, Washington, DC 20375 United States, ${ }^{2}$ The George Washington University, 2121 I Street NW, Washington, DC 20037 United States.

Nanocrystal quantum dots (QD) show great promise toward improving solar cell efficiencies through the use of quantum confinement to tune absorbance across the solar spectrum and enable multi-exciton generation. Despite this remarkable potential for high photocurrent generation, the achievable open-circuit voltage $\left(V_{\mathrm{oc}}\right)$ is fundamentally limited due to non-radiative recombination processes in QD solar cells. Here we report the highest open-circuit voltages to date for colloidal QD based solar cells under one sun illumination. This $V_{\text {oc }}$ of $692 \pm 7 \mathrm{mV}$ for $1.4 \mathrm{eV} \mathrm{PbS}$ QDs is a result of improved passivation of the defective QD surface, demonstrating $V_{\mathrm{oc}}(\mathrm{mV})=553 E_{\mathrm{g}} / q-59$ as a function of the QD bandgap $\left(E_{\mathrm{g}}\right)$. Comparing experimental $V_{o c}$ variation with the theoretical upper-limit obtained from one diode modeling of the cells with different $E_{\mathrm{g}}$, these results clearly demonstrate that there is a tremendous opportunity for improvement of $V_{\mathrm{oc}}$ to values greater than $1 \mathrm{~V}$ by using smaller QDs in QD solar cells.

$$
\text { S: }
$$
olution processability coupled with potential for multiple exciton generation processes ${ }^{1-5}$ makes nanocrystal quantum dots (QD) promising candidates for third generation low-cost and high-efficiency photovoltaics. In particular, sequential layer-by-layer assemblies of lead chalcogenide QDs (e. g., $\mathrm{PbS}^{6}, \mathrm{PbSe}^{7}$, and $\mathrm{PbSSe}^{8}$ ) have been demonstrated as an excellent photoactive absorber layer. For example, $\mathrm{PbSe}$ QD films with a bandgap $\left(E_{\mathrm{g}}\right)$ of $\sim 1.0 \mathrm{eV}$ have yielded a remarkably high short-circuit current density $\left(J_{\mathrm{sc}}\right)$ of $\sim 24 \mathrm{~mA} / \mathrm{cm}^{21}$, which is comparable to a $J_{\mathrm{sc}}$ of $24.4 \mathrm{~mA} / \mathrm{cm}^{2}$ for a nanocrystalline $S i$ absorber'. Similarly, thin-films of PbS QDs with a band gap of $\sim 1.5 \mathrm{eV}$ have also shown high $J_{\mathrm{sc}}$ values of $\sim 18 \mathrm{~mA} / \mathrm{cm}^{2},{ }^{10}$ which is greater than the $J_{\mathrm{sc}}$ of $16.75 \mathrm{~mA} / \mathrm{cm}^{2}$ obtained for amorphous $\mathrm{Si}$ solar cells ${ }^{11}$.

Despite these remarkable $J_{\mathrm{sc}}$ values ${ }^{1,7}$, the overall power conversion efficiency of these devices has been limited largely due to low open-circuit voltages $\left(V_{o c}\right)$, regardless of the type of junctions used ${ }^{12}$. Generally this is understood to be due to Fermi level pinning at the mid-gap states ${ }^{7,13}$, which are formed by a large number of surface states ${ }^{14,15}$ associated with defects on the QD surface. As a result, the barrier height between the metal and the QDs has been relatively invariant for metals with differing work functions ${ }^{7}$. This makes it difficult to create large barriers at the metal-QD Schottky junction or implement an ohmic contact to the QDs using high work function metals $(e . g ., \mathrm{Au})^{16}$.

We have fabricated Schottky junction solar cells with the highest open-circuit voltages ever reported for colloidal QD based solar cells by improving the passivation of the PbS QD surface and metal-QD interface with LiF passivation. By fitting the measured dark $J-V$ characteristics of the solar cells with different QD sizes and therefore different bandgaps, we extract various diode parameters including the saturation current and examine the effect of quantum confinement on the dark $J-\mathrm{V}$ characteristics. Combining these results with the experimental device performance under one-sun illumination, we predict that it is possible to increase the $V_{\text {oc }}$ value to over $1 \mathrm{~V}$ with smaller QDs.

\section{Results}

Several Schottky junction solar cells were fabricated (Fig 1a) using PbS nanocrystal QD films spin coated on commercial indium-tin oxide (ITO) glass. The Schottky junction was formed by evaporation of a $\mathrm{LiF}(1 \mathrm{~nm}) / \mathrm{Al}$ $(100 \mathrm{~nm})$ contact defined by a shadowmask. Full details of the device fabrication are described in Methods. The $\mathrm{PbS}$ nanocrystals were oleic acid $(\mathrm{OA})$ capped and synthesized following the procedure developed by Hines and Scholes (see Methods for full details of the synthesis and characterization) ${ }^{17}$. The bandgap of the absorber layer was controlled by changing the diameter of the PbS QDs, and the energy bandgap was determined through 
(a)

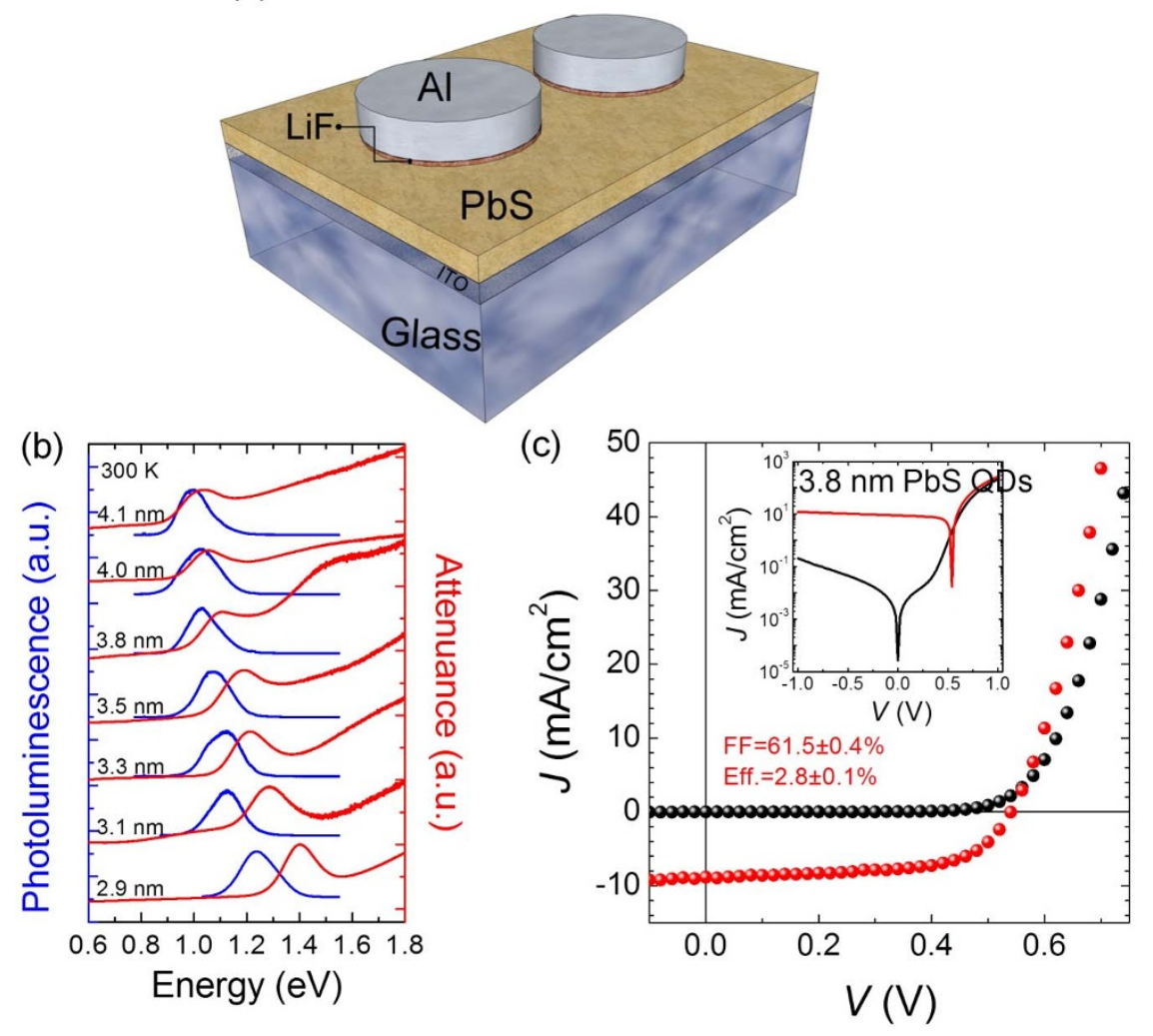

Figure 1 Surface barrier solar cells based on semiconducting PbS quantum dots and quantum confinement effects. (a) Schematic of metal-PbS QD Schottky junction solar cells (ITO/PbS QDs/LiF/Al). (b) Room-temperature photoluminescence (PL) and attenuance spectra of films assembled with OA-PbS QD with different sizes as estimated using the empirical equation by Moreels et al ${ }^{18}$. The size of QDs estimated here is in good agreement with the results obtained from TEM size distribution statistics (see Supplementary information for details). (c) Representative $J-V$ characteristics of QD solar cells in air under AM $1.5 \mathrm{G}$ filtered spectral illumination $\left(100 \mathrm{~mW} / \mathrm{cm}^{2}\right)$ with very high FF of $>60 \%$. The inset shows the $J-V$ curve in semilogarithm scale.

room-temperature photoluminescence (PL) and attenuance spectra (see Fig. 1b). As-synthesized QDs showed a well-defined firstexciton attenuance energy ranging from $1.0 \mathrm{eV}$ to $1.4 \mathrm{eV}(4.1 \mathrm{~nm}$ to $2.9 \mathrm{~nm}$ ).

Representative $J-V$ characteristics under illumination and in the dark are shown in Fig. 1c. The highest power conversion efficiency of $2.8 \pm 0.1 \%$ was obtained with a very high $\mathrm{FF}$ of $\sim 61.5 \pm 0.4 \%, V_{\mathrm{oc}}=$ $545 \pm 9 \mathrm{mV}$, and $J_{\mathrm{sc}}=8.6 \pm 0.3 \mathrm{~mA} / \mathrm{cm}^{2}$ under AM $1.5 \mathrm{G}$ filtered spectral illumination $\left(100 \mathrm{~mW} / \mathrm{cm}^{2}\right)$ for devices with $1.1 \mathrm{eV}$ PbS QD absorber layers.

The $J-V$ characteristics of devices with different bandgaps of QDs were measured under one sun in air. The $V_{\text {oc }}$ values were then plotted as a function of the QD bandgap energy as illustrated in Fig. 2, and were shown to increase linearly with QD bandgap energy $\left(E_{\mathrm{g}}\right)$ as $V_{o c}(m V)=553 E_{g} / q-59$, where $q$ is the elementary electric charge (e). In addition to the linear fit, literature $V_{\mathrm{oc}}$ values are plotted in Figure 2 for comparison. These literature values were obtained from QD solar cells based on $p$-type PbS QDs and employing three different types of junctions; (1) metal-QD Schottky junction ${ }^{6,19-23}$; (2) $p-n$ heterojunction using $n$-type wide bandgap metal oxides such as $\mathrm{TiO}_{2}$ or $\mathrm{ZnO}^{12,24-27}$, or $n$-type organic materials (C60 and PCBM $)^{28,29}$; and (3) a recently introduced $p$ - $n$ homojunction using $n$-doped $\mathrm{PbS}$ QDs $^{30}$. We obtained a $V_{\text {oc }}$ of $692 \pm 7 \mathrm{mV}$ from cells based on $2.9 \mathrm{~nm}$ PbS QDs (1.4 eV) under AM 1.5 G filtered spectral illumination $\left(100 \mathrm{~mW} / \mathrm{cm}^{2}\right)$, which is, to the best of our knowledge, the highest $V_{\mathrm{oc}}$ ever demonstrated from a colloidal QD based solar cell. In addition, for a given nanocrystal diameter, all of the $V_{\mathrm{oc}}$ values reported in Figure 2 are the highest reported for any device geometry. All solar cell performance data is summarized in Table I.
In general, the fundamental process determining $V_{\mathrm{oc}}$ is recombination, and the dark saturation current can be considered a direct measure of the recombination in the device. The relationship between dark saturation current and $V_{\mathrm{oc}}$ is inferred from the ideal diode equation under illumination ${ }^{31}$,

$$
J=J_{0}\left[\exp \left(\frac{q V}{n k T}\right)-1\right]-J_{s c}
$$

where $J_{0}$ is the dark saturation current density $\left(\mathrm{A} / \mathrm{cm}^{2}\right), J_{\mathrm{sc}}$ is the short circuit current density $\left(\mathrm{A} / \mathrm{cm}^{2}\right)$, and $n$ is the ideality factor. Assuming that the $J_{\mathrm{sc}}$ is voltage independent and $J_{\mathrm{sc}} \gg J_{0}$, the $V_{\mathrm{oc}}$ is then given by setting $J$ to 0 ,

$$
V_{o c}=\frac{n k T}{q} \ln \left(\frac{J_{s c}}{J_{0}}\right)
$$

From equation (2), $V_{\mathrm{oc}}$ is proportion to $\ln \left(J_{\mathrm{sc}} / J_{0}\right)$, and therefore $J_{0}$ needs to be as small as possible to maximize $V_{\text {oc }}$. For this reason, we measured $J-V$ curves (symbols) in the dark for devices (ITO/PbS $\mathrm{QDs} / \mathrm{LiF} / \mathrm{Al}$ ) using different sizes of $\mathrm{PbS}$ QDs, plotted as symbols in Fig. 3a. An increase in the forward dark current density was observed with increasing size, suggesting that hole injection could be enhanced due to reduction in the Schottky barrier height. This result is consistent with recent observations of the variation in barrier height between $\mathrm{Ag}$ and $\mathrm{PbS} \mathrm{QDs}$, showing that the barrier height increased for smaller QDs ${ }^{16}$. To investigate the variation in saturation current, series resistance, and shunt resistance by quantum confinement, the dark current density of the QD solar cell was fit by a one diode model including both series and shunt resistances; 


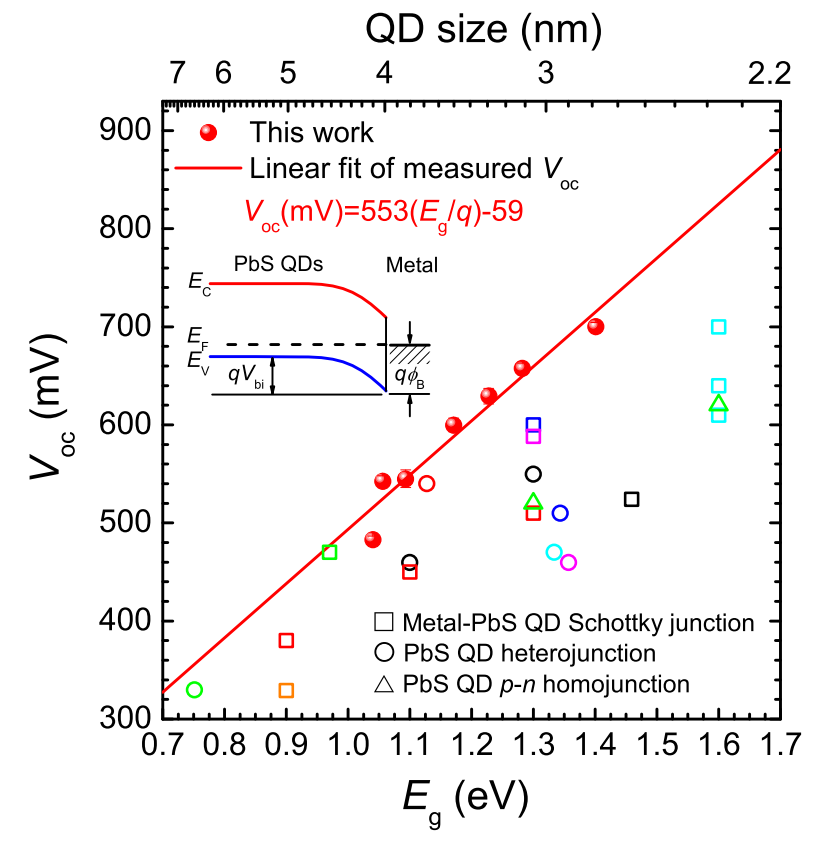

Figure $2 \mid \mathrm{PbS}$ quantum dot solar cells with high open-circuit voltages. Experimentally measured open-circuit voltage $\left(V_{\mathrm{oc}}\right)$ as a function of bandgap $\left(E_{\mathrm{g}}\right)$ in $\mathrm{PbS}$ QD solar cells under 1 sun in air (closed circle). A linear fit to measured $V_{\text {oc }}$ values yields $V_{o c}(m V)=553 E_{g} / q-59$, where $q$ is the electronic charge $(e)$. In addition to the linear fit, previously reported literature $V_{\mathrm{oc}}$ values are also plotted for comparison. These literature values were obtained from QD solar cells based on p-type PbS QDs employing three different types of junctions; (1) metal-QD Schottky junction (open circle $)^{6,19-23}$; (2) $p-n$ heterojunction using $n$-type wide bandgap metal oxides such as $\mathrm{TiO}_{2}$ or $\mathrm{ZnO}^{12,24-27}$, or $n$-type organic materials (C60 and PCBM $)^{28,29}$ (open square); and (3) a recently introduced $p-n$ homojunction using $n$-doped PbS QDs (closed triangle) ${ }^{30}$. The inset illustrates the energy-band diagram of the metal- $p$-type semiconductor junction at thermal equilibrium.

$$
J=J_{0}\left[\exp \left(\frac{q\left(V-J A R_{s}\right)}{n k T}\right)-1\right]+\frac{V-J A R_{s}}{A R_{s h}}
$$

where $J_{0}$ is the dark saturation current density $\left(\mathrm{A} / \mathrm{cm}^{2}\right), R_{\mathrm{s}}$ is the series resistance $(\Omega), R_{\mathrm{sh}}$ is the shunt resistance $(\Omega), A$ is the device area $\left(\mathrm{cm}^{2}\right)$, and $n$ is the ideality factor. This transcendental equation is solved iteratively using Newton's method to give the current density at a given bias voltage. Measured $J-V$ data in the dark is fitted by first computing the logarithm to the base 10 of the current density data, then minimizing the sum of the squared deviations of the data from the logarithm of the current density computed using equation (3). The minimization routine uses the Levenberg-Marquardt algorithm ${ }^{32}$, where a Jacobian matrix is derived from analytical expressions for the partial derivatives of $\log _{10} J$ with respect to each of the fitting variables; $J_{0}, n, R_{\mathrm{s}}$ and $R_{\mathrm{sh}}$. This method provides an efficient and reliable fitting routine, provided the initial guess for the fitted variables is close to their fitted values. As seen in Fig. 3a, the solid lines are fitted curves, which are in good agreement with measured data. The performance of all devices in the dark are summarized in Table I.

A parasitic series and shunt resistance associated with the cells was shown to be mostly size dependent. As seen in Fig. 3b, the series resistance tends to increase significantly after decreasing the size by only $1 \mathrm{~nm}$. A similar trend is also reported by Gao et al. ${ }^{16}$, as the $\mathrm{PbS}$ QD size changes from $2.4 \mathrm{~nm}$ to $6.4 \mathrm{~nm}$. Overall the series resistance depends on a number of other parameters in the devices, such as resistance of the films, contact resistance and sometimes geometrical factors $^{31}$. For these QD systems, it has been reported that size dependence of carrier mobility is responsible for the increased series resistance in $\mathrm{PbS}$ QD films as decreasing size ${ }^{16,33}$.In addition, an increased series resistance is attributed to larger contact resistance to smaller QDs. This is because the contact resistance depends on the barrier height ${ }^{31}$, while the barrier height tends to increase for smaller $\mathrm{QDs}^{16}$. It is interesting to note that the shunt resistance increased by almost two orders of magnitude for smaller QDs (Fig. 3c). Although the quantum confinement effect on the shunt resistance is not well known, an increased shunt resistance may be attributed to dense and pin-hole free multilayered assemblies with smaller $\mathrm{QDs}^{34}$, which potentially reduces the leakage across the junction. The quantum confinement effect on parasitic series and shunt resistances associated with QD solar cells is still under investigation.

It is important to note that several factors could contribute to the improving $V_{\mathrm{oc}}$ values seen in this study. First, the thickness of the $\mathrm{LiF}$ interfacial layer was carefully optimized ${ }^{35}$ prior to the $\mathrm{Al}$ deposition onto the PbS QD layers. When $10 \AA$ thick LiF was deposited between the $\mathrm{PbS}$ QDs and the $\mathrm{Al}$, the $J_{0}$ decreased by one order of magnitude compared to a device with $6 \AA$ thick LiF. Further increasing the LiF thickness up to $14 \AA$ reduced the $J_{0}$ but at the expense of increasing the series resistances. These results suggest that the optimized LiF thickness is critical for reducing $J_{0}$ by passivating localized traps in the PbS QDs close to the junction ${ }^{12,22,25,29,36}$, thus suppressing nonradiative recombination processes in the cells. Further details are discussed in Supplementary information. Second, a sequential layer-by-layer spin-coating process in air was used to fabricate the devices, resulting in oxidation and a concomitant passivation effect on the PbS QD surface that eliminates trap centers ${ }^{22,29,37}$. The X-ray photoelectron spectroscopy (XPS) confirmed the presence of multiple oxide compounds in the actual device samples fabricated in air (refer to Figure S3 in Supplementary information for detailed XPS data $)^{29,37}$. In fact, Zhao et al. ${ }^{29}$ report that a smaller recombination rate constant was observed for their air-annealed PbS QD devices, confirming that recombination processes are significantly suppressed due to passivation effects by some degree of oxidation, consequently leading to the highest $V_{\text {oc }}$ of $0.47 \mathrm{~V}$, compared with $\mathrm{N}_{2}$-annealed, pristine, and ozone-treated devices ${ }^{29}$.

\begin{tabular}{|c|c|c|c|c|c|c|c|c|}
\hline $\begin{array}{l}d \\
(\mathrm{~nm})\end{array}$ & $\begin{array}{c}t \\
(\mathrm{~nm})\end{array}$ & $\begin{array}{c}J_{\mathrm{sc}} \\
\left(\mathrm{mA} / \mathrm{cm}^{2}\right)\end{array}$ & $\begin{array}{l}V_{o c} \\
(\mathrm{mV})\end{array}$ & $\begin{array}{l}\mathrm{FF} \\
(\%)\end{array}$ & $\begin{array}{l}\text { Eff. } \\
\text { (\%) }\end{array}$ & $\begin{array}{c}J_{0} \\
\left(\mathrm{~A} / \mathrm{cm}^{2}\right)\end{array}$ & $\begin{array}{c}R_{\mathrm{s}} \cdot A \\
\left(\Omega \cdot \mathrm{cm}^{2}\right)\end{array}$ & $\begin{array}{c}R_{\mathrm{sh}} \cdot A \\
\left(\Omega \cdot \mathrm{cm}^{2}\right)\end{array}$ \\
\hline 2.9 & 148 & $5.7 \pm 0.5$ & $692 \pm 7$ & $34.9 \pm 2.8$ & $1.4 \pm 0.1$ & $(1.7 \pm 0.7) \times 10^{-10}$ & $383 \pm 4.2$ & $(3.6 \pm 0.2) \times 10^{6}$ \\
\hline 3.1 & 151 & $6.7 \pm 0.8$ & $657 \pm 6$ & $45.4 \pm 0.9$ & $2.1 \pm 0.1$ & $(3.7 \pm 2.1) \times 10^{-10}$ & $54 \pm 11$ & $(1.2 \pm 0.1) \times 10^{6}$ \\
\hline 3.3 & 198 & $8.6 \pm 0.8$ & $629 \pm 8$ & $48.4 \pm 1.8$ & $2.6 \pm 0.2$ & $(6.6 \pm 2.1) \times 10^{-10}$ & $16 \pm 5.7$ & $(2.9 \pm 0.3) \times 10^{6}$ \\
\hline 4.0 & 115 & $8.3 \pm 0.3$ & $542 \pm 5$ & $48.6 \pm 1.1$ & $2.2 \pm 0.2$ & $(2.2 \pm 0.9) \times 10^{-8}$ & $6.2 \pm 0.1$ & $(1.0 \pm 0.1) \times 10^{4}$ \\
\hline 4.1 & 148 & $8.4 \pm 0.7$ & $483 \pm 3$ & $59.7 \pm 1.3$ & $2.5 \pm 0.3$ & $(5.1 \pm 0.8) \times 10^{-8}$ & $1.0 \pm 0.1$ & $(1.6 \pm 0.4) \times 10^{4}$ \\
\hline
\end{tabular}


(a)

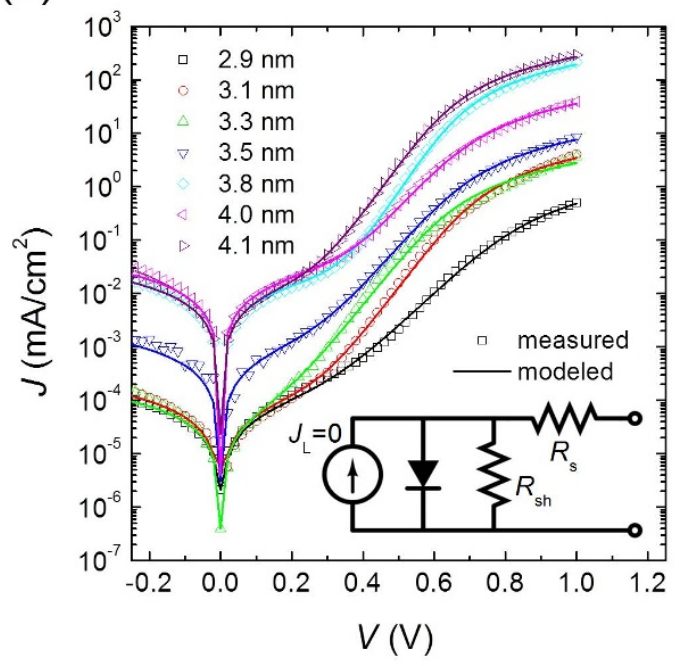

(d)

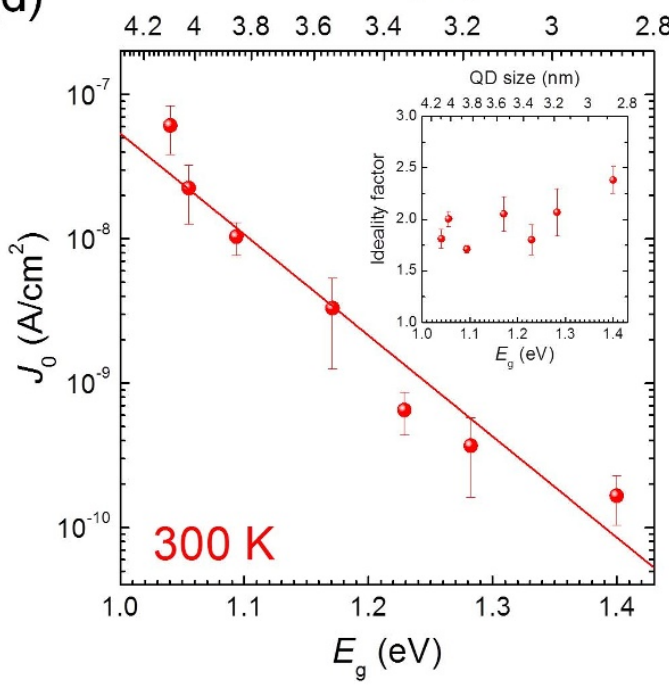

(b)
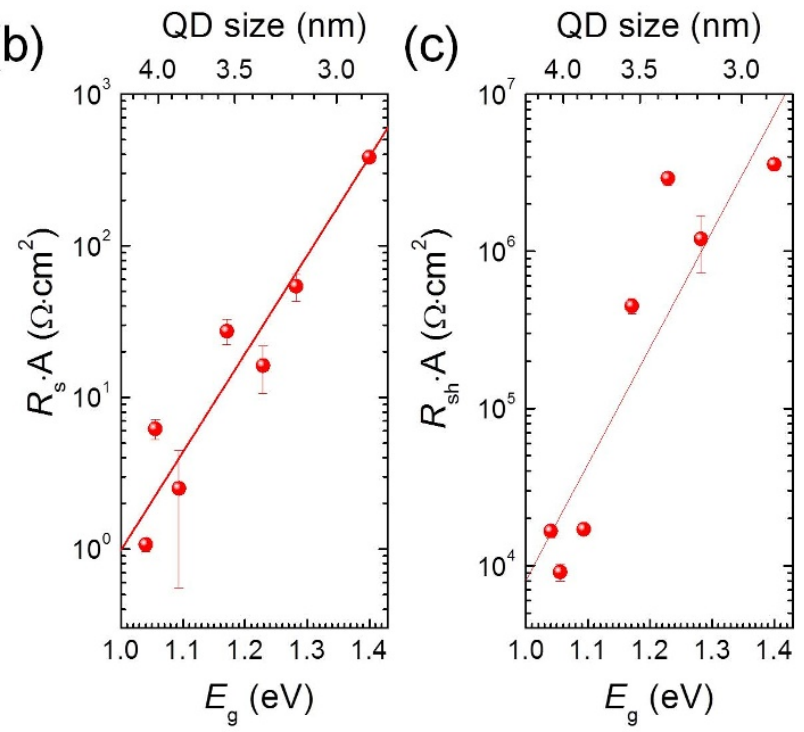

(e)

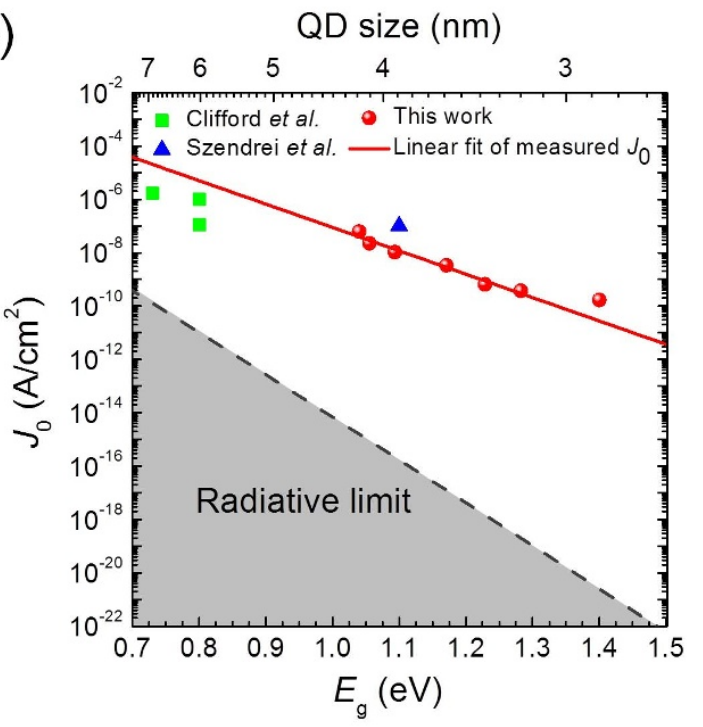

Figure $3 \mid$ Quantum confinement effect in dark current-voltage characteristics of PbS QD solar cells. (a) Current density-voltage ( $J-V$ ) characteristics in the dark for PbS nanocrystal QD Schottky-barrier solar cells with the structure of ITO/PbS QDs/LiF/Al and a varying QD size from 2.9 nm to 4.1 nm. The open symbols are the experimentally measured data, while the solid lines are fits according to a one diode model including series and shunt resistances (equation 3). The inset shows the equivalent circuit of the diode in the dark. (b-d) The quantum confinement effect on diode parameters, (b) series resistance $\left(R_{\mathrm{s}}\right),(\mathrm{c})$ shunt resistance $\left(R_{\mathrm{sh}}\right)$, and $(\mathrm{d})$ saturation current density $\left(J_{0}\right)$. $A$ is the diode area $\left(\mathrm{cm}^{2}\right)$. The inset of Fig. $3 \mathrm{~d}$ shows the ideality factor $(n)$ vs QD bandgap energy $\left(E_{\mathrm{g}}\right)$. (e) Calculated limit of the $J_{0}$ as a function of $E_{\mathrm{g}}$ and size in metal-PbS QD junction. The dash line indicates the radiative limit of $J_{0}$ calculated using equation (7). The literature values (square ${ }^{39}$ and triangle ${ }^{40}$ ) in $J_{0}$ for metal-PbS QD Schottky junction are shown for comparison.

From the measured and modeled $J-V$ characteristics, $J_{0}$ and $n$ were plotted as a function of QD bandgap energy. A strong quantum confinement effect in $J_{0}$ was observed in Figure 3d, showing that $J_{0}$ decreases by three orders of magnitude for a size decrease of only $1 \mathrm{~nm}$. The ideality factors were varied between 1.7 and 2.4, showing no clear correlation with varying size (inset of Fig. 3d). The lower limit of $J_{0}$ is a function of bandgap $\left(E_{\mathrm{g}}\right)^{38}$,

$$
J_{0}=J_{00} \exp \left(-\frac{E_{g}}{n k T}\right)
$$

where $J_{00}$ is a prefactor $\left(\mathrm{A} / \mathrm{cm}^{2}\right)$ which depends on the current transport mechanism of the device ${ }^{31}$. From the slope and the intercept of the linear fit of experimental data using $\ln J_{0}=\ln J_{00}-E_{g} / n k T, J_{00}$ and $n$ were estimated $51.6 \pm 6.2 \mathrm{~A} / \mathrm{cm}^{2}$ and 1.92 , respectively. Using equation (4) combined with the obtained values in $J_{00}$ and $n$, variations in $J_{0}$ across a wide range of bandgaps (from $0.7 \mathrm{eV}$ to $1.5 \mathrm{eV}$ ) is predicted, as illustrated in Fig. 3e. Other experimental literature values of $J_{0}$ are also plotted for comparison (Fig. 3e) ) $^{39,40}$, showing similar trends. For comparison we also show the radiative limit for $J_{0}$ (i. e., the ideal solar cell material).

\section{Discussion}

In the thermodynamic limit, the dark current density is limited only by radiative recombination and the EQE is unity for above bandgap photons and zero for all other photons. The dark current density is given by the generalized Planck formula

$$
J_{\text {rad }}=\frac{2 \pi q}{h^{3} c^{2}} \int_{E_{g}}^{\infty} \frac{E^{2}}{\exp [(E-q V / k T)]-1} d E
$$


Here we have assumed the limiting optical arrangement, such that the device has a perfect mirror on one face and a vacuum on the other face $^{41}$. In the Boltzmann approximation, the -1 in the denominator of this expression can be neglected and the integral then has a simple analytical form, allowing the radiative current density to be written as

$$
J_{\text {rad }}=J_{0, \text { rad }} \exp \left(\frac{q V}{k T}\right)
$$

where the radiative saturation current density $\left(J_{0, \mathrm{rad}}\right)$ is

$$
J_{0, \text { rad }}=\frac{2 \pi q}{h^{3} c^{2}} k T\left(E_{g}^{2}+2 k T E_{g}+2(k T)^{2}\right) \exp \left(-\frac{E_{g}}{k T}\right)
$$

$J_{0 \text {,rad }}$ was plotted as a function of bandgap using equation (7) in Fig. 3e. The difference between the measured $J_{0}$ for QD solar cells and the $J_{0, \text { rad }}$ calculated when the radiative recombination process is dominant allows us to determine the theoretical upper limit of the $V_{\text {oc }}$ available for device improvement. This assumes all non-radiative recombination processes in the metal-PbS QD Schottky junction solar cells are suppressed ${ }^{25,29,36,37}$. For example, the use of core/shell ${ }^{42}$ or core/shell/shell ${ }^{43}$ nanocrystal QDs is expected to improve the $V_{\mathrm{oc}}$ by reducing a large number of traps associated with surface defects, greatly suppressing the non-radiative recombination contributions to the dark current.

Finally, we have analyzed the upper limit to the $V_{\mathrm{oc}}$ achievable with the experimentally measured $J_{0}$ values of the devices, using equation (2) with the ideality factor of 1.92 at $300 \mathrm{~K}$. In this case, the limiting photocurrent was calculated under the ASTM AM1.5 G spectrum as a function of the QD bandgap, again assuming an external quantum efficiency (EQE) of unity for above bandgap

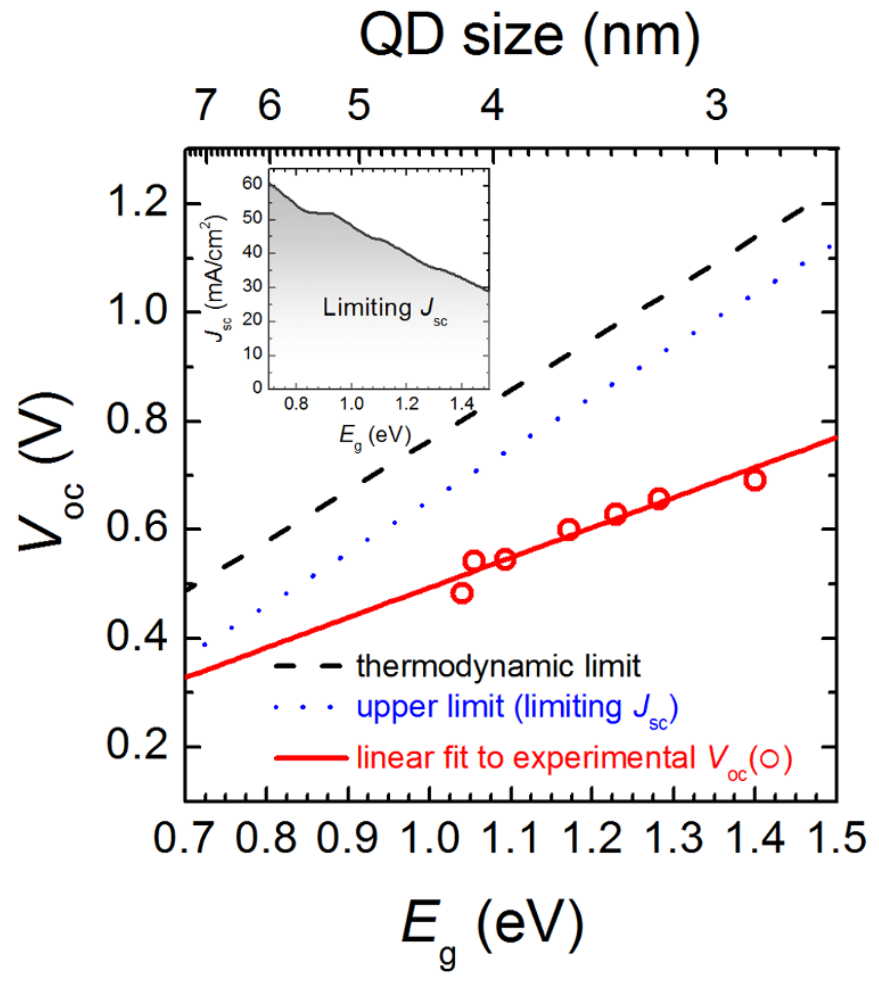

Figure $4 \mid$ Limit in the open-circuit voltage in metal-QD Schottky junction solar cells. The limit of the $V_{\mathrm{oc}}$ as a function of the bandgap ( $E_{\mathrm{g}}$ ) of PbS QDs: the thermodynamic limit (dash) of $V_{\text {oc }}$ calculated using limiting $J_{\mathrm{sc}}$ and $J_{0}$, respectively (Eq. 9); the upper limit (dot) of $V_{\mathrm{oc}}$ calculated using limiting $J_{\mathrm{sc}}$ and experimentally measured $J_{0}$ (Eq. 10); the experimentally measured $V_{\mathrm{oc}}$ (solid) as a function of $\mathrm{PbS}$ QD size. The inset shows the limiting $J_{\mathrm{sc}}$ in thermodynamic limit as a function of $E_{\mathrm{g}}$ under AM1.5 G. photons. In this case, the short-circuit current density is given by

$$
J_{s c}=\frac{q}{h c} \int_{0}^{\lambda g} \lambda I(\lambda) d \lambda
$$

where $I(\lambda)$ is the spectral irradiance in units of $\mathrm{W} \mathrm{m}^{-2} \mathrm{~nm}^{-1}$ and the bandgap wavelength is given by $\lambda_{g}=h c / E_{g}$. Thus, the upper limit of $V_{\text {oc }}$ is:

$$
V_{o c, \text { upper limit }}=\frac{1.92 k T}{q} \ln \left(\frac{\frac{q}{h c} \int_{0}^{\lambda_{g}} \lambda I(\lambda) d \lambda}{J_{00} \exp \left(-\frac{E_{g}}{1.92 k T}\right)}\right)
$$

where $J_{00}$ is $51.6 \pm 6.2 \mathrm{~A} / \mathrm{cm}^{2}$ at $300 \mathrm{~K}$. Similarly, we calculated the thermodynamic limit for the $V_{\text {oc }}$ by substituting $J_{0, \text { rad }}$ (Eq. 7 ) and the limiting $J_{\text {sc }}$ obtained from equation 8 into equation (2):

$V_{\text {oc,thermodynamic limit }}=\frac{k T}{q} \ln \left(\frac{\frac{q}{h c} \int_{0}^{\lambda_{g}} \lambda I(\lambda) d \lambda}{\frac{2 \pi q}{h^{3} c^{2}} k T\left(E_{g}^{2}+2 k T E_{g}+2(k T)^{2}\right) \exp \left(-\frac{E_{g}}{k T}\right)}\right)_{(10)}$

In Figure 4, these two limits for $V_{\text {oc }}$ values are plotted along with the experimentally measured $V_{\mathrm{oc}}$ values and the associated linear fit of $V_{o c}=553 E_{g} / q-59$ in the range of $0.7 \mathrm{eV}$ to $1.5 \mathrm{eV}$. As discussed earlier, the difference between the thermodynamic and upper limit for $V_{\mathrm{oc}}$ is due to the increased $J_{0}$ resulting from the non-radiative recombination processes in metal-QD junction solar cells. Comparing experimental $V_{\text {oc }}$ values with the calculated upper limit of $V_{\mathrm{oc}}$ is a good indicator for assessing how much enhanced photocurrents could further improve the $V_{\mathrm{oc}}$ of $\mathrm{PbS} \mathrm{QD}$ solar cells. These results clearly suggest that there is substantial opportunity for $V_{\mathrm{oc}}$ enhancement by increasing $J_{\mathrm{sc}}$ in this system. For example, the highest $V_{\text {oc }}$ of $1129 \mathrm{mV}$ can be achievable at a bandgap of $1.5 \mathrm{eV}$ (i.e., $2.6 \mathrm{~nm}$ PbS QDs), which is about $92 \%$ of the thermodynamic limit. One way to enhance the $J_{\text {sc }}$ values could be to implement front semitransparent metal contacts with anti-reflective coatings in metal-QD Schottky junctions, enabling thicker absorber layers to be used. In devices reported to date, the $J_{\text {sc }}$ of the device is limited by the relatively thin active QD absorber layer thickness, a consequence of Schottky junction formation at the back metal contact (e.g. Al, LiF/ $\mathrm{Al}, \mathrm{Mg} / \mathrm{Al}$ ) rather than at the front contact (e.g. ITO).

To summarize, we report enhanced open-circuit voltages as a function of the QD bandgap for metal-PbS QD Schottky junction solar cells as a result of reduced non-radiative recombination processes. Under one sun illumination, we demonstrate the highest $V_{\mathrm{oc}}$ of $692 \pm 7 \mathrm{mV}$ (1.4 eV PbS QDs) ever reported for colloidal QD based solar cells. By analyzing the diode parameters extracted from the measured dark $J-V$ characteristics, we find a strong quantum confinement effect on the saturation current density, which decreases by three orders of magnitude with only a $1 \mathrm{~nm}$ reduction in QD size. The parasitic series and shunt resistances associated with the devices are also shown to be strongly size dependent. Using the measurement of the size dependent saturation current and the shortcircuit current calculated from a detailed balance model, the upper limit of the open-circuit voltage achievable for QD sizes varying in size from $2.6 \mathrm{~nm}$ to $7.4 \mathrm{~nm}$ is presented. By comparing experimental $V_{\mathrm{oc}}$ values with this upper limit, we suggest that it is possible to enhance the open-circuit voltage to greater than $1 \mathrm{~V}$ by using smaller QDs to minimize the short-circuit current losses in Schottky-barrier solar cells.

\section{Methods}

Material. Lead oxide (PbO, 99.9+\%), oleic acid (OA, 90\%), 1-octadecene (ODE, $90 \%$ ), hexamethyldisilathiane (TMS, synthesis grade), hexanes (98.5\%) and acetone (99.9\%) were purchased from Sigma Aldrich. Ethanol (200 proof) was purchased from the Warner Graham Company. All chemicals were used as received. 
PbS nanocrystal QD synthesis. The synthesis of $\mathrm{PbS}$ nanocrystal QDs followed the procedure developed by Hines and Scholes ${ }^{17}$, and was performed under inert conditions using standard Schlenk techniques. ODE $(20 \mathrm{~mL})$ was dried and degassed by heating to $110^{\circ} \mathrm{C}$ under vacuum for $1 \mathrm{hr}$, then TMS $(658 \mu \mathrm{L})$ was added to create a stock solution of $151 \mathrm{mM}$ TMS in ODE. In a typical synthesis, $376 \mathrm{mg} \mathrm{PbO}, 1.4 \mathrm{~g} \mathrm{OA}$ and $11.8 \mathrm{~g}$ of ODA were mixed in a $50 \mathrm{~mL}$ three neck flask and heated to $110^{\circ} \mathrm{C}$ under vacuum for 30 minutes to create $\mathrm{Pb}$ (oleate) $)_{2}$. The flask was purged with $\mathrm{Ar}$ and heated to an injection temperature between $130^{\circ} \mathrm{C}$ and $150^{\circ} \mathrm{C}$. Next, $5 \mathrm{~mL}$ of TMS stock solution was injected into the $\mathrm{Pb}$ (oleate) ${ }_{2}$ solution instantly turning the color black. After several minutes, the reaction was cooled by placing the flask in liquid nitrogen. To separate the QDs from unreacted reagents and byproducts, $2 \mathrm{~mL}$ of hexane and $10 \mathrm{~mL}$ of acetone and/or ethanol were added to the reaction mixture which was then centrifuged at $4000 \mathrm{rpm}$ for $4 \mathrm{~min}$. The precipitated QDs formed a black solid at the bottom of the centrifuge vial and the supernatant was removed by decanting. The QDs were washed one more time with hexane and acetone and/or ethanol, dried with $\mathrm{Ar}$, and stored in a glove box. The reaction yield is nearly $100 \%$ relative to TMS. The mass of $\mathrm{PbS}$ in the final product was determined using a TA Instruments Q500 thermogravimetric analyzer (TGA). The QD diameter was varied by changing a combination of the injection temperature, growth time, and oleic acid to lead ratio (see Tables S1 and S2 in the Supplementary Information for full details of the synthesis).

PbS nanocrystal QD characterization. Attenuance spectra of the PbS QD films were obtained at room temperature using a Fourier Transform Infrared (FTIR) spectrometer (VERTEX $80 \mathrm{v}$, Bruker Optics) equipped with a $\mathrm{CaF}_{2}$ beamsplitter and a DLaTGS detector. Samples were prepared by drop casting onto glass slides, and a portion of the substrate which was not coated with the PbS film was used as a reference. The samples were evacuated to 1.6 Torr in the sample chamber in order to minimize oxidation of the $\mathrm{PbS}$ surface and to suppress $\mathrm{H}_{2} \mathrm{O}$ and $\mathrm{CO}_{2}$ absorption features in the spectra.

The PL spectra of films of PbS QDs drop cast onto silicon wafers were obtained at room temperature with a triple spectrometer (Trivista 557, Princeton Instruments) utilizing a $\mathrm{LN}_{2}$ cooled InGaAs linear array detector (OMA V, Princeton Instruments). The PL samples were mounted in an Oxford Instruments cryostat equipped with a $\mathrm{ZnS}$ window and pumped to $\sim 10^{-5}$ mTorr. The samples were mounted in the cryostat in order to keep them under vacuum and minimize oxidation of the $\mathrm{PbS}$ surface and suppress absorption features in the spectra from $\mathrm{H}_{2} \mathrm{O}$ and $\mathrm{CO}_{2}$.

TEM samples were prepared by making dilute chloroform solutions and drop casting a small volume $(<500 \mu \mathrm{L})$ onto lacey carbon TEM grids. TEM images were obtained on a JEOL $2200 \mathrm{~F} 3$ transmission electron microscope operating at $200 \mathrm{kV}$. The CCD camera on which the images were recorded was calibrated with a gold lattice magnification standard.

X-ray photoelectron spectroscopy (XPS) (K-Alpha XPS system, Thermo Fisher Scientific Inc.) was performed in a commercial UHV system (typically $1 \times 10^{-9}$ Torr base pressure) equipped with an $\mathrm{Al} \mathrm{K} \alpha$ micro-focused monochromator.

X-ray power refraction (XRD) data was obtained on a Rigaku Smartlab instrument operating in parallel beam mode and indexed to bulk PbS by comparison to JCPDS card\#1-880. Samples were prepared by drop casting concentrated $\mathrm{CHCl}_{3}$ solutions of the $\mathrm{PbS}$ nanocrystals onto quartz substrates followed by solvent evaporation. For assynthesized PbS QDs, the position of the PbS QD XRD diffraction peak is consistent with bulk $\mathrm{PbS}$ (refer to Figure $\mathrm{S} 1$ in Supplementary information).

Device fabrication. A sequential layer-by-layer spin-coating procedure was conducted as reported in the literature ${ }^{6}$ to prepare the PbS QD active layers. Briefly, QD films were deposited onto pre-cleaned ITO-coated glass substrate by spin-coating $\sim 5 \mathrm{mg} / \mathrm{ml}$ solution of PbS-OA in chloroform at $4000 \mathrm{rpm}$ for $30 \mathrm{~s}$. The PbS QD films were then immersed in $20 \mathrm{mM} \mathrm{1,3-benzenedithiol} \mathrm{(BDT,} \mathrm{99 \% ,} \mathrm{Aldrich)} \mathrm{in}$ acetonitrile for $30 \mathrm{~s}$, followed by spin-coating at $4000 \mathrm{rpm}$ for $30 \mathrm{~s}$ to remove residual solution (see Figure S4 in Supplementary information). This process was repeated until the desired film thickness had been achieved. All procedures were performed in air. Finally, the devices were completed by shadowmask evaporation of a $\operatorname{LiF}(1 \mathrm{~nm}) /$ $\mathrm{Al}(100 \mathrm{~nm})$ top contact at $10^{-7}$ torr. Six devices were made on each substrate, each device with an active area of $0.11 \mathrm{~cm}^{2}$.

Electrical characterization of devices. The current density-voltage $(J-V)$ measurements were performed with a parameter analyzer (Agilent 4156C) in air without any encapsulation under the spectral output from a $150 \mathrm{~W}$ solar simulator (Newport Corporation) using an AM $1.5 \mathrm{G}$ filter. The irradiance $\left(100 \mathrm{~mW} / \mathrm{cm}^{2}\right)$ of the solar simulator was adjusted using a standard Si photodetector (818-SL-L, Newport Corporation) that had been cross-calibrated by a reference Si cell traceable to the National Renewable Energy Laboratory (NREL).

1. Semonin, O. E. et al. Peak External Photocurrent Quantum Efficiency Exceeding $100 \%$ via MEG in a Quantum Dot Solar Cell. Science 334, 1530-1533 (2011).

2. Schaller, R. D. \& Klimov, V. I. High efficiency carrier multiplication in PbSe nanocrystals: Implications for solar energy conversion. Phys. Rev. Lett. 92 (2004)

3. Ellingson, R. J. et al. Highly Efficient Multiple Exciton Generation in Colloidal PbSe and PbS Quantum Dots. Nano Lett. 5, 865-871 (2005).

4. Sandberg, R. L. et al. Multiexciton Dynamics in Infrared-Emitting Colloidal Nanostructures Probed by a Superconducting Nanowire Single Photon Detector. ACS Nano (2012).
5. Cunningham, P. D. et al. Enhanced Multiple Exciton Generation in Quasi-OneDimensional Semiconductors. Nano Lett. 11, 3476-3481 (2011).

6. Szendrei, K., Gomulya, W., Yarema, M., Heiss, W. \& Loi, M. A. PbS nanocrystal solar cells with high efficiency and fill factor. Appl. Phys. Lett. 97, 203501-203503 (2010).

7. Luther, J. M. et al. Schottky Solar Cells Based on Colloidal Nanocrystal Films. Nano Lett. 8, 3488-3492 (2008).

8. Ma, W., Luther, J. M., Zheng, H., Wu, Y. \& Alivisatos, A. P. Photovoltaic Devices Employing Ternary PbSxSe1-x Nanocrystals. Nano Lett. 9, 1699-1703 (2009)

9. Yamamoto, K. et al. in MRS Spring Meeting.

10. Tang, J. et al. Colloidal-quantum-dot photovoltaics using atomic-ligand passivation. Nat Mater 10, 765-771 (2011).

11. Benagli, S. et al. in 24th European Photovoltaic Solar Energy Conference.

12. Gao, J. et al. n-Type Transition Metal Oxide as a Hole Extraction Layer in PbS Quantum Dot Solar Cells. Nano Lett. 11, 3263-3266 (2011).

13. Pal, B. N. et al. High-Sensitivity p-n Junction Photodiodes Based on PbS Nanocrystal Quantum Dots. Adv. Funct. Mater. 22, 1741-1748 (2012).

14. Fu, H. \& Zunger, A. InP quantum dots: Electronic structure, surface effects, and the redshifted emission. Physical Review B 56, 1496-1508 (1997).

15. Califano, M., Franceschetti, A. \& Zunger, A. Temperature Dependence of Excitonic Radiative Decay in CdSe Quantum Dots: The Role of Surface Hole Traps. Nano Lett. 5, 2360-2364 (2005).

16. Gao, J. et al. Quantum Dot Size Dependent J-V Characteristics in Heterojunction $\mathrm{ZnO} / \mathrm{PbS}$ Quantum Dot Solar Cells. Nano Lett. 11, 1002-1008 (2011).

17. Hines, M. A. \& Scholes, G. D. Colloidal PbS Nanocrystals with Size-Tunable NearInfrared Emission: Observation of Post-Synthesis Self-Narrowing of the Particle Size Distribution. Adv. Mater. 15, 1844-1849 (2003).

18. Moreels, I. et al. Size-Dependent Optical Properties of Colloidal PbS Quantum Dots. ACS Nano 3, 3023-3030 (2009).

19. Debnath, R. et al. Ambient-Processed Colloidal Quantum Dot Solar Cells via Individual Pre-Encapsulation of Nanoparticles. J. Am. Chem. Soc. 132, 5952-5953 (2010).

20. Fu, H. et al. Impact of the Growth Conditions of Colloidal PbS Nanocrystals on Photovoltaic Device Performance. Chem. Mater. 23, 1805-1810 (2011).

21. Johnston, K. W. et al. Schottky-quantum dot photovoltaics for efficient infrared power conversion. Appl. Phys. Lett. 92, 151115-151113 (2008)

22. Tang, J. et al. Schottky Quantum Dot Solar Cells Stable in Air under Solar Illumination. Adv. Mater. 22, 1398-1402 (2010)

23. Tang, J. et al. Quantum Dot Photovoltaics in the Extreme Quantum Confinement Regime: The Surface-Chemical Origins of Exceptional Air- and Light-Stability. ACS Nano 4, 869-878 (2010).

24. Wang, X. et al. Enhanced Open-Circuit Voltage in Visible Quantum Dot Photovoltaics by Engineering of Carrier-Collecting Electrodes. ACS Applied Materials \& Interfaces 3, 3792-3795 (2011).

25. Brown, P. R. et al. Improved Current Extraction from $\mathrm{ZnO} / \mathrm{PbS}$ Quantum Dot Heterojunction Photovoltaics Using a MoO3 Interfacial Layer. Nano Lett. 11, 2955-2961 (2011).

26. Pattantyus-Abraham, A. G. et al. Depleted-Heterojunction Colloidal Quantum Dot Solar Cells. ACS Nano 4, 3374-3380 (2010).

27. Luther, J. M. et al. Stability Assessment on a 3\% Bilayer PbS/ZnO Quantum Dot Heterojunction Solar Cell. Adv. Mater. 22, 3704-3707 (2010).

28. Klem, E. J. D. et al. Planar PbS quantum dot/C[sub 60] heterojunction photovoltaic devices with 5.2\% power conversion efficiency. Appl. Phys. Lett. 100, 173109-173104 (2012)

29. Zhao, N. et al. Colloidal PbS Quantum Dot Solar Cells with High Fill Factor. ACS Nano 4, 3743-3752 (2010).

30. Tang, J. et al. Quantum Junction Solar Cells. Nano Lett. 12, 4889-4894 (2012)

31. Sze, S. M. Physics of Semiconductor Devices. 2nd edn, (John Wiley \& Sons, Inc. 1981)

32. Press, W. H., Flannery, B. P., Teukolsky, S. A. \& Vetterling, W. T. Numerical Recipes in C. 2nd edn, (Cambridge University Press, 1992).

33. Liu, Y. et al. Dependence of Carrier Mobility on Nanocrystal Size and Ligand Length in PbSe Nanocrystal Solids. Nano Lett 10, 1960-1969 (2010).

34. Hanrath, T., Choi, J. J. \& Smilgies, D.-M. Structure/Processing Relationships of Highly Ordered Lead Salt Nanocrystal Superlattices. ACS Nano 3, 2975-2988 (2009).

35. Brabec, C. J., Shaheen, S. E., Winder, C., Sariciftci, N. S. \& Denk, P. Effect of LiF/ metal electrodes on the performance of plastic solar cells. Appl. Phys. Lett. 80, 1288-1290 (2002).

36. Barkhouse, D. A. R., Pattantyus-Abraham, A. G., Levina, L. \& Sargent, E. H. Thiols Passivate Recombination Centers in Colloidal Quantum Dots Leading to Enhanced Photovoltaic Device Efficiency. ACS Nano 2, 2356-2362 (2008).

37. Hardman, S. J. O. et al. Electronic and surface properties of $\mathrm{PbS}$ nanoparticles exhibiting efficient multiple exciton generation. PCCP 13, 20275-20283 (2011).

38. Green, M. A. Solar Cells: Operating Principles, Technology, and System Applications. (The University of New South Wales, 1998).

39. Clifford, J. P., Johnston, K. W., Levina, L. \& Sargent, E. H. Schottky barriers to colloidal quantum dot films. Appl. Phys. Lett. 91, 253117-253113 (2007).

40. Szendrei, K. et al. Exploring the Origin of the Temperature-Dependent Behavior of PbS Nanocrystal Thin Films and Solar Cells. Adv. Funct. Mater. 22, 1598-1605 (2012). 
41. Letay, G. \& Bett, A. W. in 17th European Photovoltaic Solar Energy Conference (Munuch, Germany, 2001).

42. Dabbousi, B. O. et al. (CdSe)ZnS Core-Shell Quantum Dots: Synthesis and Characterization of a Size Series of Highly Luminescent Nanocrystallites. The Journal of Physical Chemistry B 101, 9463-9475 (1997).

43. Talapin, D. V. et al. CdSe/CdS/ZnS and CdSe/ZnSe/ZnS Core-Shell-Shell Nanocrystals. The Journal of Physical Chemistry B 108, 18826-18831 (2004).

\section{Acknowledgements}

This research was performed while W. Yoon and D. Placencia held a National Research Council (NRC) Research Associateship Awards (RAP) at the U.S. Naval Research Laboratory. The Office of Naval Research (ONR) is acknowledged for financial support of this work.

\section{Author contributions}

W.Y. and J.G.T. conceived and designed the experiment. W.Y. carried out the experiments. J.E.B., D.P. and E.E.F. synthesized the materials. M.P.L conducted the theoretical study. W.Y. wrote the manuscript. All authors discussed the results and reviewed the manuscript.

\section{Additional information}

Supplementary information accompanies this paper at http://www.nature.com/ scientificreports

Competing financial interests: The authors declare no competing financial interests. How to cite this article: Yoon, W.J. et al. Enhanced Open-Circuit Voltage of PbS Nanocrystal Quantum Dot Solar Cells. Sci. Rep. 3, 2225; DOI:10.1038/srep02225 (2013).

(c) (1) $(9)$ This work is licensed under a Creative Commons AttributionNonCommercial-NoDerivs 3.0 Unported license. To view a copy of this license, visit http://creativecommons.org/licenses/by-nc-nd/3.0 